\title{
Eddy-current analysis using vector hysteresis models with play and stop hysterons
}

\author{
$\operatorname{AUTHOR}(\mathrm{S})$ : \\ Matsuo, T; Osaka, Y; Shimasaki, M
}

\section{CITATION:}

Matsuo, T ...[et al]. Eddy-current analysis using vector hysteresis models with play and stop hysterons. IEEE TRANSACTIONS ON MAGNETICS 2000, 36(4): 1172-1177

\section{ISSUE DATE:}

2000-07

URL:

http://hdl.handle.net/2433/39989

\section{RIGHT:}

(c)2000 IEEE. Personal use of this material is permitted. However, permission to reprint/republish this material for advertising or promotional purposes or for creating new collective works for resale or redistribution to servers or lists, or to reuse any copyrighted component of this work in other works must be obtained from the IEEE. 


\title{
Eddy-Current Analysis Using Vector Hysteresis Models with Play and Stop Hysterons
}

\author{
Tetsuji Matsuo, Member, IEEE, Yoshihiro Osaka, and Masaaki Shimasaki, Member, IEEE
}

\begin{abstract}
Vector hysteresis models are applied to an eddy-current analysis. The vector hysteresis models are composed by play hysterons and stop hysterons. The eddy-current analysis shows that both play and stop hysteron models can effectively describe isotropic vector hysteretic behavior. The stop hysteron model is more efficient in analyzes using the magnetic vector potential than the play model because the stop model can give the magnetic field from the magnetic flux density without an iteration process.
\end{abstract}

Index Terms-Asymmetrical magnetization, eddy-current analysis, play hysteron, stop hysteron, vector hysteresis.

\section{INTRODUCTION}

$\mathbf{T}$ HE PREISACH model [1] is a powerful tool for describing various hysteretic characteristics, including the vector property [2]. In finite element analyzes, however, the Preisach model has several difficulties such as a large memory requirement and high computation costs to calculate the magnetic field $H$ from the magnetic flux density $B$ [3].

Recently, some alternative hysteresis models [3]-[7] have been proposed, which seem efficient enough to be applied to the finite element analysis. However, most of these models are scalar models, even though the electromagnetic field analysis with ferromagnetic materials needs 2-D or 3-D vector hysteretic properties [8], [9].

The present paper, firstly constructs 2-D vector hysteresis models using the play and stop hysteron models [5], [7], and secondly applies them to a 2-D eddy-current analysis. The play hysteron model can be implemented more simply than the Preisach model, and it has properties equivalent to those of the scalar static Preisach model [10], [7]. The stop hysteron model is as simple as the play hysteron model and is suitable for calculating $H$ from $B$.

\section{Play and Stop Hysteron Models}

\section{A. Play Hysteron Model}

The scalar play hysteron model [5], [7] describes the hysteretic relation between $H$ and $B$ as

$$
B(H)=\sum_{k=1}^{M} f_{k}\left(p_{k}(H)\right)
$$

Manuscript received October 25, 1999.

T. Matsuo and M. Shimasaki are with the Graduate School of Engineering, Kyoto University, Kyoto, 606-8501, Japan (e-mail: \{tmatsuo; simasaki\}@kuee.kyoto-u.ac.jp).

Y. Osaka is with the Graduate School of Energy Science, Kyoto University, Kyoto, 606-8501, Japan.

Publisher Item Identifier S 0018-9464(00)06838-2.

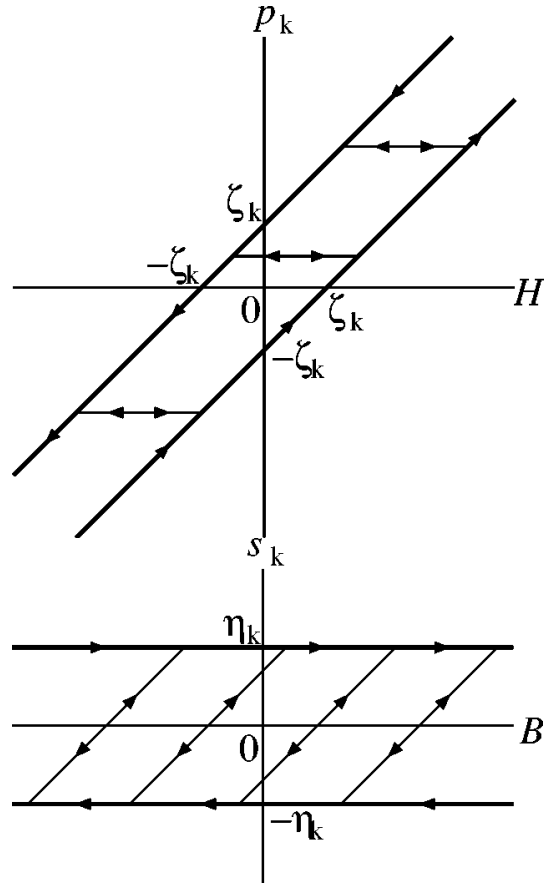

Fig. 1. Hysteron operators.

where

$M \quad$ is the number of hysteron operators,

$p_{k} \quad$ is the play hysteron operator, and

$f_{k} \quad$ is a single-valued function.

The play hysteron operator $p_{k}$ is given by

$$
p_{k}(H)=\max \left(\min \left(p_{k}^{0}, H+\zeta_{k}\right), H-\zeta_{k}\right)
$$

where $p_{k}^{0}$ is the value of $p_{k}$ at the previous time-point, and $\zeta_{k}$ is a constant. The characteristics of operator $p_{k}$ are illustrated in Fig. 1(a).

The play hysteron model, described by (1) and (2), can be less cumbersome in numerical implementations than the Preisach model, and it can describe hysteretic characteristics equivalently to the scalar static Preisach model [10], [7].

\section{B. Stop Hysteron Model}

The hysteretic characteristics given by the scalar stop hysteron model are written as

$$
\begin{aligned}
H(B) & =\sum_{k=1}^{M} g_{k}\left(s_{k}(B)\right) \\
s_{k}(B) & =\max \left(\min \left(B-B^{0}+s_{k}^{0}, \eta_{k}\right),-\eta_{k}\right)
\end{aligned}
$$

where

$g_{k}$ is a single-valued function, 


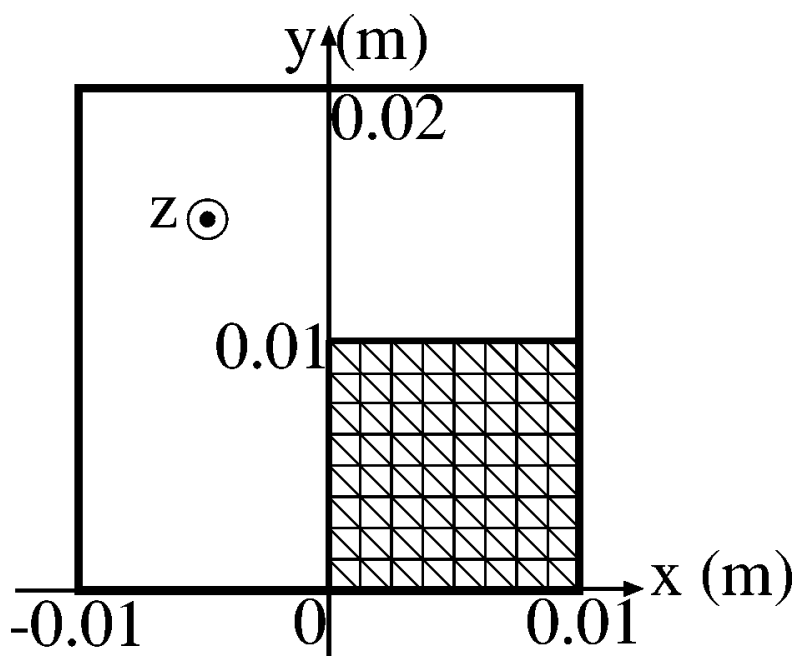

Fig. 2. Analyzed iron-core.

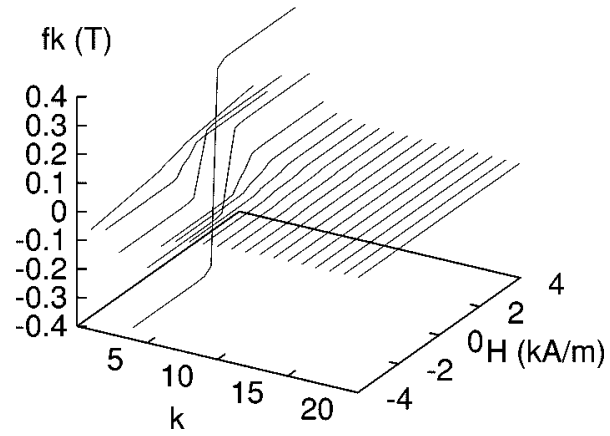

Fig. 3. Functions $f_{k}$.

$s_{k} \quad$ is the stop hysteron,

$B^{0} s_{k}^{0}$ are the values at the previous time-point, and

$\eta_{k} \quad$ is a constant.

The characteristics of operator $s_{k}$ are illustrated in Fig. 1(b).

While this model is as simple as the play hysteron model, it is suitable for calculating $H$ from $B$ because the stop operator makes clockwise hysteresis loops, as in Fig. 1(b).

If $H(B)$ becomes a single-valued function when $|B|>B_{\text {sat }}$ ( $B_{\text {sat }}$ : saturation magnetic flux density), $B^{0}$ and $s_{k}^{0}$ should be restricted within $\left|B^{0}\right| \leq B_{\text {sat }}$ and $\left|s_{k}^{0}\right| \leq \min \left(B_{\text {sat }}, \eta_{k}\right)$, respectively.

Since the derivatives of functions (2) and (4) are easily obtained, both play and stop hysteron models can be used effectively together with the Newton method.

\section{VECTOR HySTERESIS MODELS}

\section{A. Vector Hysteresis Model by Play Hysterons}

Mayergoyz [2] has proposed a 2-D vector hysteresis model as a superposition of scalar models.

$$
\boldsymbol{B}(\boldsymbol{H})=\int_{0}^{\pi} \boldsymbol{e}_{\varphi} B_{\varphi}\left(\boldsymbol{e}_{\varphi} \cdot \boldsymbol{H}\right) d \varphi \simeq \frac{\pi}{N} \sum_{k=0}^{N-1} \boldsymbol{e}_{\varphi k} B_{\varphi k}\left(\boldsymbol{e}_{\varphi k} \cdot \boldsymbol{H}\right)
$$

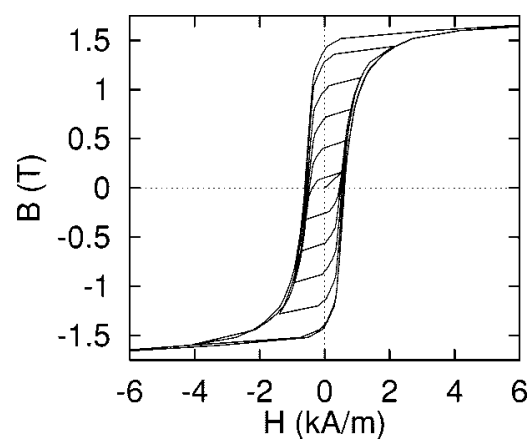

(a)

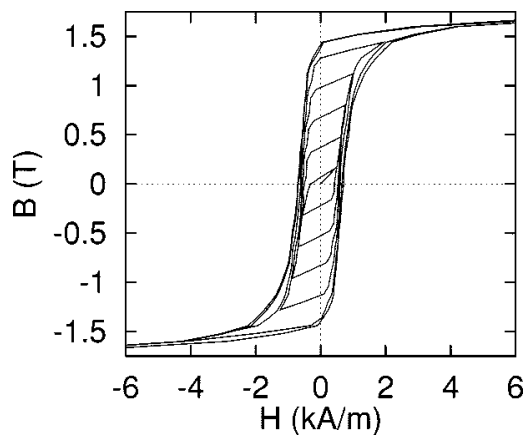

(b)

Fig. 4. Hysteretic characteristics.

where

$B_{\varphi}$ is a scalar hysteretic function for the $\varphi$-direction,

$e_{\varphi} \quad$ is the unit vector in the $\varphi$-direction,

$N \quad$ is the number of angular divisions, and

$\varphi_{k} \quad=\pi k / N$.

The present paper uses the play hysteron model to represent the function $B_{\varphi}\left(H_{\varphi}\right)$, where $H_{\varphi}=\boldsymbol{e}_{\varphi} \cdot \boldsymbol{H}$. Since the play hysteron model is equivalent to the scalar static Preisach model, the identification method for the vector Preisach model in [2] can be applied to this vector model.

The electromagnetic analysis using magnetic vector potential requires the inverse function of (5) that yields $H=(H x, H y)$ from a known vector $\boldsymbol{B}=(B x, B y)$. The inversion is given by solving (6) for $\boldsymbol{H}$ by the Newton method.

$$
\left[\begin{array}{c}
B x \\
B y
\end{array}\right]=\frac{\pi}{N} \sum_{k=0}^{N-1}\left[\begin{array}{c}
\cos \varphi_{k} \\
\sin \varphi_{k}
\end{array}\right] B_{\varphi k}\left(H x \cos \varphi_{k}+H y \sin \varphi_{k}\right)
$$

The Newton method requires the Jacobian matrix of (6).

$$
\frac{\partial \boldsymbol{B}}{\partial \boldsymbol{H}}=\frac{\pi}{N} \sum_{k=0}^{N-1} \frac{\mathrm{d} B_{\varphi k}}{\mathrm{~d} H_{\varphi k}}\left[\begin{array}{cc}
\cos ^{2} \varphi_{k} & \cos \varphi_{k} \sin \varphi_{k} \\
\cos \varphi_{k} \sin \varphi_{k} & \sin ^{2} \varphi_{k}
\end{array}\right]
$$

The inverse matrix of $\partial B / \partial \boldsymbol{H}$ gives $\partial \boldsymbol{H} / \partial B$.

$$
\partial \boldsymbol{H} / \partial \boldsymbol{B}=(\partial \boldsymbol{B} / \partial \boldsymbol{H})^{-1}
$$

Equations (7) and (8) lead to

$$
\partial H x / \partial B y=\partial H y / \partial B x \text {. }
$$




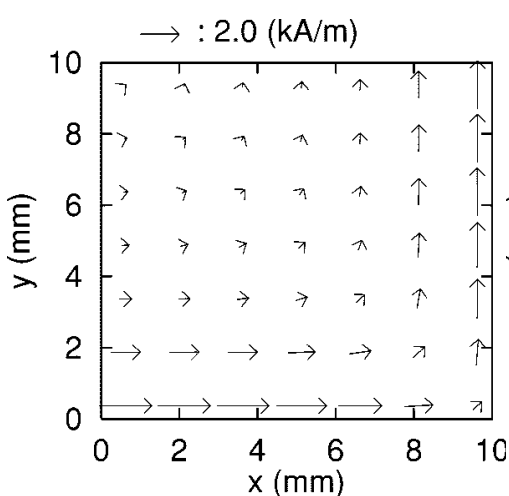

(a)

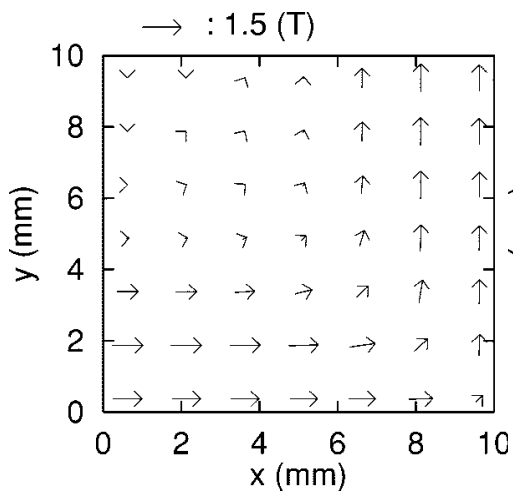

(d)

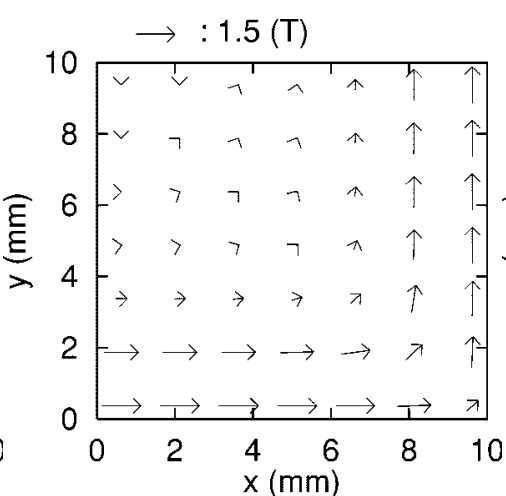

(b)

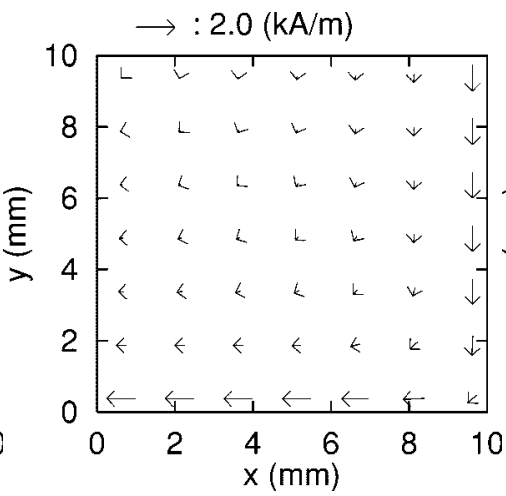

(e)

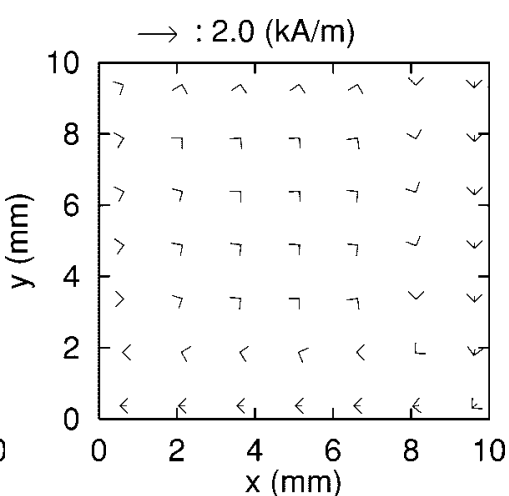

(c)

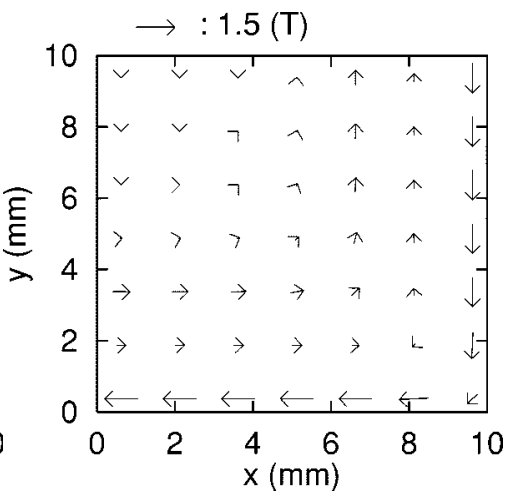

(f)

Fig. 5. $\boldsymbol{H}$ - and $\boldsymbol{B}$-fields at $t=7,12,17 \mathrm{msec}$ by play model.

\section{B. Vector Hysteresis Model by Stop Hysterons}

A 2-D vector function $\boldsymbol{H}(\boldsymbol{B})$ is constructed similarly as a superposition of scalar stop hysteron models.

$$
\boldsymbol{H}(\boldsymbol{B})=\frac{\pi}{N} \sum_{k=0}^{N-1} \boldsymbol{e}_{\varphi k} H_{\varphi k}\left(\boldsymbol{e}_{\varphi k} \cdot \boldsymbol{B}\right)
$$

where $H_{\varphi k}$ is a scalar hysteretic function by the stop hysteron model for the $\varphi_{k}$-direction. This vector model also has the property of (9).

\section{EDDY-CURRENT ANALYSIS}

The 2-D eddy-current analysis is carried out by using the magnetic vector potential $A$. The magnetic field $(H x, H y)$ is written as a function of $(B x, B y)$.

$$
H x=H x(B x, B y), \quad H y=H y(B x, B y)
$$

where $B x=\partial A / \partial y$ and $B y=-\partial A / \partial x$.

The equation for the eddy-current analysis is then given by

$$
\frac{\partial}{\partial x} H y(B x, B y)-\frac{\partial}{\partial y} H x(B x, B y)=-\sigma\left(\frac{\partial A}{\partial t}+\frac{\partial \phi}{\partial z}\right)
$$

where $\phi$ is the electric scalar potential.

The Galerkin finite element method and the backward Euler time difference scheme lead to (13) from (12).

$\left\{F\left(\left\{A^{\mathrm{k}}\right\}\right)\right\}=\left\{K\left(\left\{A^{\mathrm{k}}\right\}\right)\right\}+[M] \frac{\left\{A^{\mathrm{k}}\right\}-\left\{A^{\mathrm{k}-1}\right\}}{\Delta t}+\{J\}=0$

$$
\begin{aligned}
K_{i} & =\sum_{e} \iint_{(e)}\left(-H y \frac{\partial N_{i}}{\partial x}+H x \frac{\partial N_{i}}{\partial y}\right) d x d y, \\
M_{i j} & =\sum_{e} \iint_{(e)} \sigma N_{i} N_{j} d x d y \\
J_{i} & =\sum_{e} \iint_{(e)} \sigma \frac{\partial \phi}{\partial z} N_{i} d x d y
\end{aligned}
$$

where

$N_{i} \quad$ is the interpolation function,

$\Delta t \quad$ is the time-step, and

the superscripts of $A$ indicate the time-points.

Equation (13) is solved for the unknown vector $\left\{A^{\mathrm{k}}\right\}$ by the Newton method. The Newton method requires the Jacobian matrix $\partial\{F\} / \partial\{A\}$ given by

$$
\begin{aligned}
& {\left[\frac{\partial\{F\}}{\partial\{A\}}\right]_{i j}} \\
& =\frac{M_{i j}}{\Delta t}+\sum_{e} \iint_{(e)}\left\{\left(\frac{\partial H x}{\partial B x} \frac{\partial N_{j}}{\partial y}-\frac{\partial H x}{\partial B y} \frac{\partial N_{j}}{\partial x}\right) \frac{\partial N_{i}}{\partial y}\right. \\
& \left.\quad-\left(\frac{\partial H y}{\partial B x} \frac{\partial N_{j}}{\partial y}-\frac{\partial H y}{\partial B y} \frac{\partial N_{j}}{\partial x}\right) \frac{\partial N_{i}}{\partial x}\right\} d x d y .
\end{aligned}
$$

The matrix $\partial\{F\} / \partial\{A\}$ becomes symmetric due to (9), (14), and (15). 


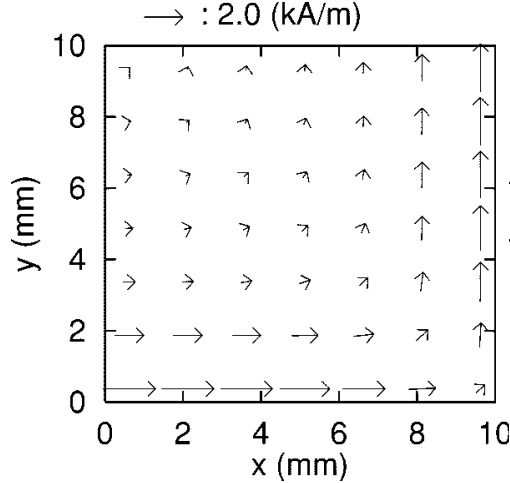

(a)

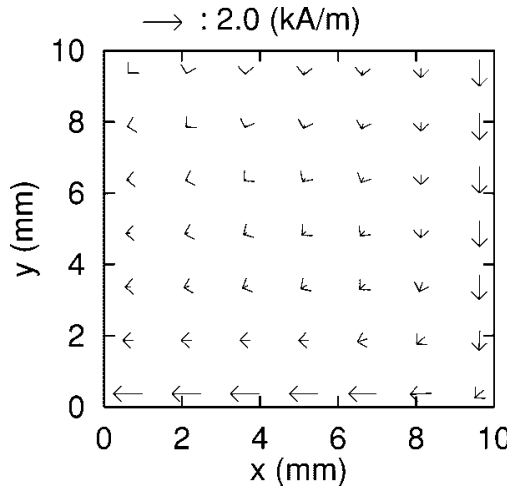

(c)

Fig. 6. $\quad \boldsymbol{H}$ - and $\boldsymbol{B}$-fields at $t=7,17 \mathrm{msec}$ by stop model.

\section{EXAMPLES OF EDDY-CURRENT ANALYSIS}

The iron-core with a square cross section shown in Fig. 2 is analyzed. The alternating external electric field is imposed in the $z$-direction as

$$
\partial \phi / \partial z=-E_{z 0} \sin 2 \pi f t
$$

where $E_{z 0}=1 \mathrm{~V} / \mathrm{m}$ and $f=50 \mathrm{~Hz}$. The electrical conductivity $\sigma$ is set at $10^{6} \mathrm{~S} / \mathrm{m}$. Before applying the electric field, the iron-core is demagnetized.

One-fourth of the core region is divided into 3200 equal triangular meshes to apply the finite element method with first-order triangular elements.

The present paper assumes isotropic hysteresis. The functions $f_{k}(k=1, \cdots, M)$ in the play hysteron model for all the angular directions is set as in Fig. 3, where $M=20$ and $\zeta_{k}=160(k-1) \mathrm{A} / \mathrm{m}$. Fig. 4(a) shows the hysteretic characteristics constructed by the play hysteron model where $N=12$. The vector model by stop hysterons are composed so as to approximately represent the inverse of the vector characteristics by the play hysteron model. Fig. 4(b) shows the hysteretic characteristics constructed by the stop hysteron model where $N=12$, $M=20, B_{\mathrm{sat}}=1.6 \mathrm{~T}, \eta_{1} \rightarrow \infty$, and $\eta_{k}=0.08(M-k+1)$ $\mathrm{T}(k=2, \cdots, M)$.

Fig. 5 shows the $\boldsymbol{H}$-and $\boldsymbol{B}$-fields by the play hysteron model at $t=7,12,17 \mathrm{msec}$, which are given from (13) with a $\Delta t$ of $0.1 \mathrm{msec}$. Fig. 5 illustrates that the eddy current prevents or delays the variations in the magnetic field. Remanence is observed in Fig. 5(d) when $\boldsymbol{H}$ is almost zero as in Fig. 5(c). The remanence is also seen in Fig. 5(f) where $\boldsymbol{B}$ around the center part of

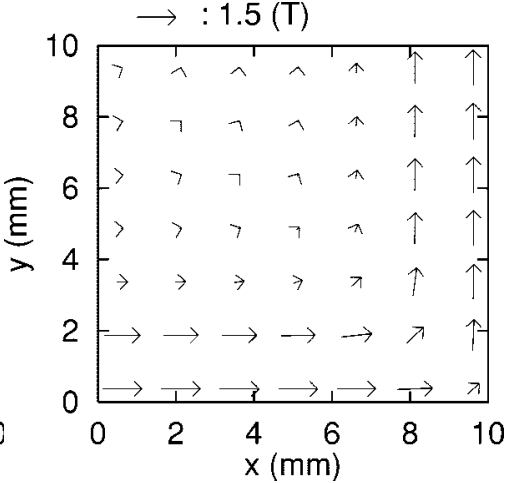

(b)

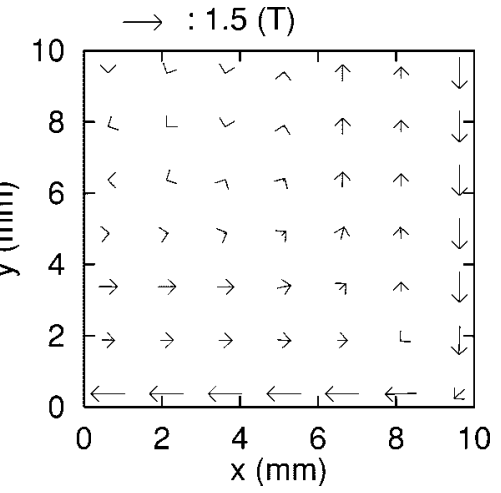

(d)

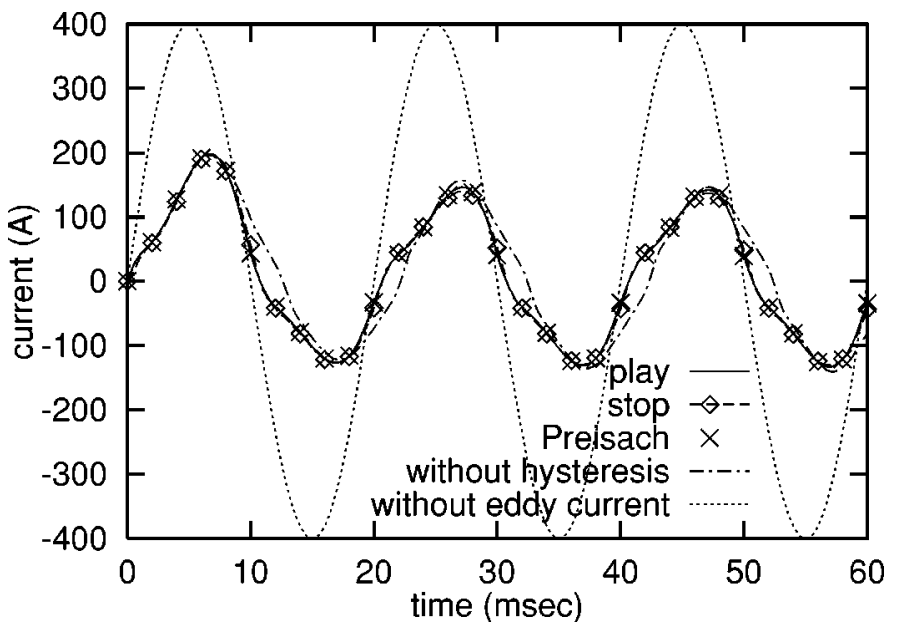

Fig. 7. Core-current.

the core is in the opposite direction of $\boldsymbol{B}$ near the core-surface and also in the opposite direction of $\boldsymbol{H}$. The vector model by play hysterons needs several Newton iterations to compute $\boldsymbol{H}$ from $\boldsymbol{B}$. The average number of Newton iterations is less than 3 to solve (6) in this analysis.

Fig. 6 shows the $\boldsymbol{H}$ - and $\boldsymbol{B}$-fields by the stop hysteron model at $t=7,17 \mathrm{msec}$, which are given from (13) with a $\Delta t$ of 0.2 msec. Figs. 5 and 6 show that the stop hysteron model gives almost the same results as the play hysteron model without the iteration process for the calculation of $\boldsymbol{H}$ from $\boldsymbol{B}$.

Fig. 7 shows the electric current in the iron-core which is the sum of the imposed current by $\partial \phi / \partial z$ and the induced eddy 


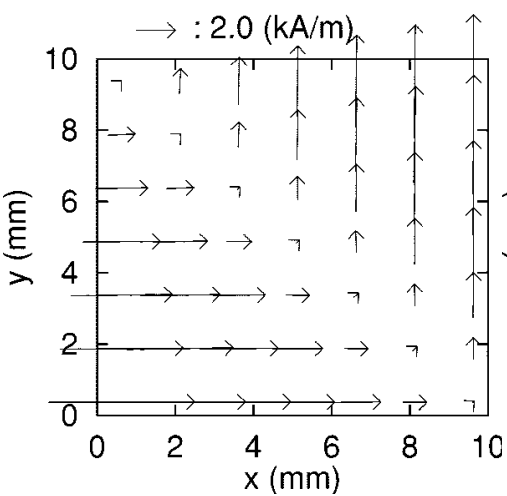

(a)

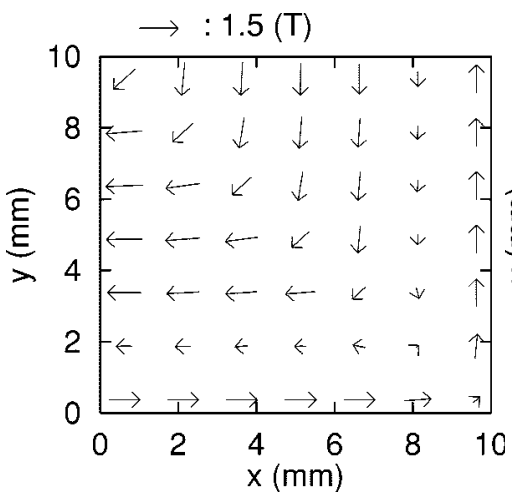

(d)

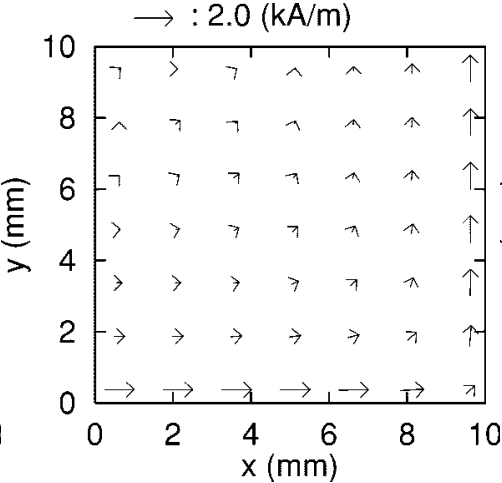

(b)

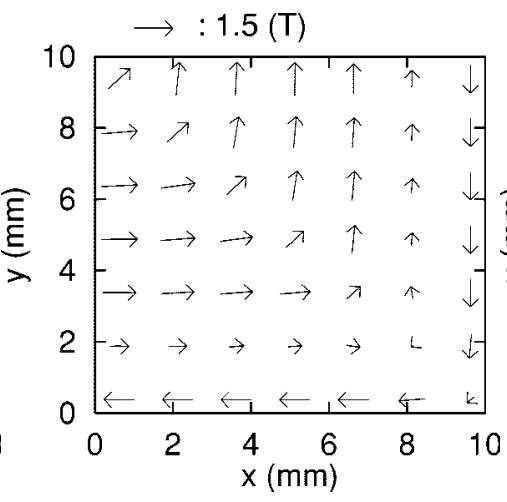

(e)

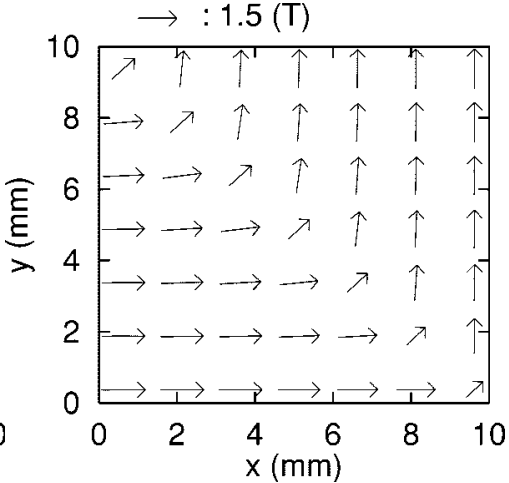

(c)

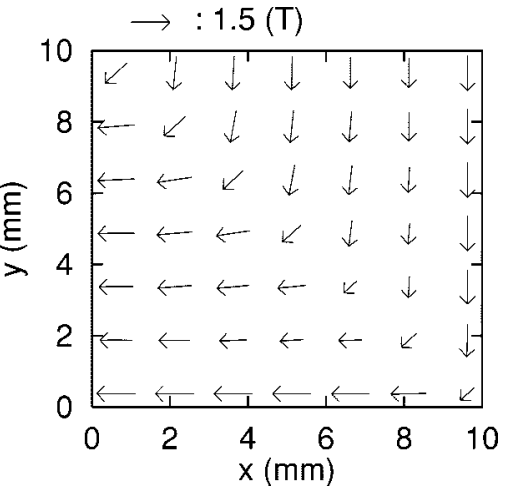

(f)

Fig. 8. $\boldsymbol{H}$ - and $\boldsymbol{B}$-fields at $t=7,17 \mathrm{msec}$ after dc excitations.

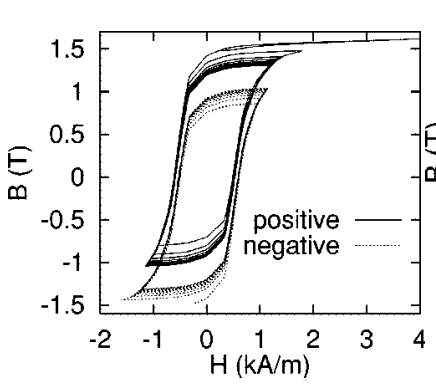

(a)

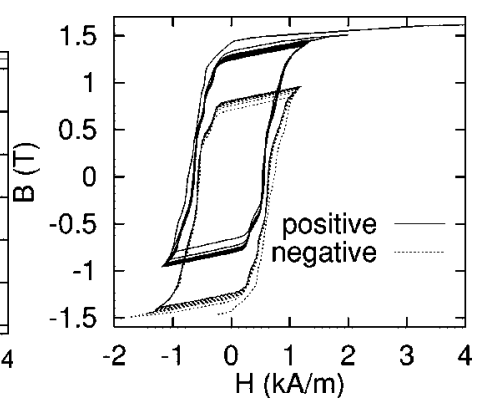

(b)

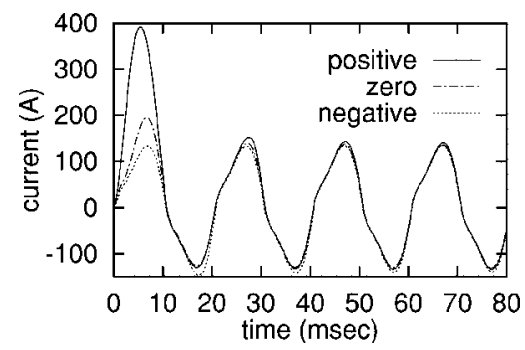

(c)

Fig. 9. Effects of dc excitations.

current. The dotted line in Fig. 7 shows the current given by the magnetostatic analysis without eddy current, while the dashdotted line is given by the nonhysteretic analysis where the initial magnetizing curve is used for the $\boldsymbol{H}-\boldsymbol{B}$ relation. The initial magnetizing curve is yielded by setting $p_{k}^{0}=0$ in the play hysteron model. For comparison, the current given by the vector Preisach model [2] is also shown in Fig. 7.
Fig. 7 shows that the eddy current reduces and delays the variation in the core-current. The maximal difference in the core-current between the cases with and without hysteresis is more than $70 \mathrm{~A}$, whereas the discrepancy between the play and stop hysteron models is less than $15 \mathrm{~A}$. As expected, the play hysteron model works equivalently to the Preisach model within a discrepancy of $10^{-3} \mathrm{~A}$.

Next, the effect of asymmetrical magnetization is examined. A large direct field of $10 \mathrm{~V} / \mathrm{m}$ or $-10 \mathrm{~V} / \mathrm{m}$ is applied and removed before imposing the alternating field. Fig. 8 shows the $\boldsymbol{H}$ - and $\boldsymbol{B}$-fields with the stop hysteron model after the dc excitations. The counter-clockwise and clockwise $\boldsymbol{B}$-fields clearly remain, as seen in Fig. 8(c) and (d), respectively, because of the dc excitations that also greatly affect the $\boldsymbol{H}$-fields, as in Fig. 8(a) and (b). Fig. 9(a) and (b) show the $H x-B x$ hysteresis loops at $(x, y)=(1.5 \mathrm{~mm}, 1 \mathrm{~mm})$ after the positive- and negative-dc excitations. Fig. 9(a) and (b) show that the positive dc excitation leads to a large transient oscillation of the magnetic field, and that the effect of dc excitations still remains in the steady state. Fig. 9(c) shows the effect of dc excitations on the core-current, which indicates that the positive dc excitation leads to a large inrush current.

\section{CONCLUSIONS}

The vector hysteresis models using play and stop hysterons are able to describe the vector hysteretic behavior effectively in an eddy-current analysis. The play hysteron model yields $\boldsymbol{H}$ from $\boldsymbol{B}$ with several Newton iterations, whereas the stop hysteron model gives $\boldsymbol{H}$ without the iteration process. 


\section{REFERENCES}

[1] F. Preisach, "Über die Magnetische Nachwirkung," Zeitschrift für Physik, vol. 94, pp. 277-302, 1935.

[2] I. D. Mayergoyz, Mathematical Models of Hysteresis. New York, NY: Springer-Verlag, 1991.

[3] N. Takahashi, S. Miyabara, and K. Fujiwara, "Problems in practical finite element analysis using Preisach hysteresis model," IEEE Trans. Magn., vol. 35, pp. 1243-1246, 1999.

[4] G. S. Park, S. Y. Hahn, K. S. Lee, and H. K. Jung, "Implementation of hysteresis characteristics using the Preisach model with $M-B$ variables," IEEE Trans. Magn., vol. 29, pp. 1542-1545, 1993.

[5] M. A. Krasnosel'skii and A. V. Pokrovskii, Systems with Hysteresis. Berlin Heidelberg: Springer-Verlag, 1989.
[6] S. Bobbio and G. Marrucci, "A possible alternative to Preisach's mode of static hysteresis," Il Nuovo Cimento, vol. 15-D, pp. 723-733, 1993.

[7] S. Bobbio, G. Miano, C. Serpico, and C. Visone, "Models of magnetic hysteresis based on play and stop hysterons," IEEE Trans. Magn., vol. 33, pp. 4417-4426, 1997.

[8] M. Enokizono, T. Todaka, and S. Kanao, "Two-dimensional magnetic properties of silicon steel sheet subjected to a rotating field," IEEE Trans. Magn., vol. 29, pp. 3550-3552, 1993.

[9] M. Enokizono and N. Noda, "Direct magnetic loss analysis by FEM considering vector magnetic properties," IEEE Trans. Magn., vol. 34, pp. 3008-3011, 1998

[10] M. Brokate, "Some mathematical properties of the Preisach model for hysteresis," IEEE Trans. Magn., vol. 25, pp. 2922-2924, 1989. 